INTERNATIONAL JOURNAL OF SYSTEMATIC BACTERIOLOGY

Vo1. 20, No. 4 October 1970

pp. $535-538$

Copyright 1970, Iowa State University Press

\title{
RELATIONSHIPS OF METABOLIC PRODUCTS TO TAXONOMY OF ANAEROBIC BACTERIA
}

\author{
W. E. C. Moore
}

Anaerobe Laboratory, V.P.I., Blacksburg, Virginia 24061

\begin{abstract}
Acid and aclohol products are extremely useful for identification of anaerobic bacteria. The types and relative amounts of products are similar from strain tostrain (eitherfresh isolatesor historic reference strains); reproducible from culture to culture within a strain; and easily determined by chromatography. Among the many different patterns of products, some, by definition, are shared by all species of the genus (e.g. Propionibacterium) and some are produced by only a single described species (e.g. Ramibacterium alactolyticum).
\end{abstract}

Historically, the family Lactobacillaceae and the genus Propionibacterium were defined on the basis of the metabolic products of the bacteria assigned to these taxa. Lactic acid was determined by chemical isolation, and propionic and acetic acids were determined using distillation procedures.

With the introduction of chromatography, analysis for these and many other metabolic products has become much more accurate. Analyses have now been made for the alcohols and acids produced by many strains representing different species in ait of the recognized genera of anaerobic bacteria.

It has become increasingly evident that metabolic products do represent a firm basis upon which to differentiate taxa, and one that is all the more useful now that analytical procedures are rapid and simple enough to be used in routine identification.

The metabolic products are very similar among the strains within each species. All tested strains of Clostridium tetani, for example, have a distinctive pattern of acid and alcohol products; large quantities of butanol, acetic and butyric acids, and small quantities of propionic acid. Every strain that we have examined, including those that have been lyophilized in collections for many years, those that have been stored as spore suspensions in dry soil, and freshly isolated strains, produced the same pattern of products. That the products correlate well with the other important characteristics of this species such as morphology, toxigenicity and biochemical properties, is evident. The same repeatability is found among strains of each species of anaerobes that has been examined. Thus, the major metabolic products appear to represent a stable property of bacterial species.

There is excellent repeatability from culture to culture within strains. Essentially the same relative proportions of products are produced from week to week or year to year with a given strain. Poor growth of a culture generally results in decreased total amounts of 
the products rather than changes in relative proportions. The patterns remain clearly recognizable. Variations between different lots of culture medium produce no detectable differences in the fermentation product patterns. Similar patterns are usually produced from a range of substrates representing the same class of compounds. If an organism can metabolize glucose, the products from fructose, galactose, maltose, sucrose, and similar carbohydrates that are attacked are essentially identical. With some species, products from lactate or pyruvate differ from those produced from carbohydrates, and this metabolic activity may further differentiate species.

Many species not fermenting carbohydrates produce distinctive patterns of alcohol and acid products from peptone or other proteina ceous media. We know of approximately 20 different patterns of alcohol and acid products. Individual patterns are characteristic either of a genus, a group of species, or an individual species.

All species of Lactobacillus produce lactic acid as the single major acid froduct from carbohydrates, and sometines from peptone - (Fig. 1). It is easily detected and mea sured by liquid-liquid chromatography of one $\mathrm{ml}$. of culture, the analysis taking about 25 minutes. Heterofermentative strains cultured under anaerobic conditions also produce small amounts of acetic acid, while homofermentative strains produce trace amounts of acetic acid or none. Bifidobacteria all produce major amounts of acetic and lactic acids, always with equal or greater amounts of acetic acid. Actinomyces species produce major amounts of succinic acid, with lesser amounts of acetic, formic, and lactic acids. The relative amounts of lactic and succinic acids vary with the amount of carbonate or $\mathrm{CO}_{2}$ in the medium. With increased levels of $\mathrm{CO}_{2}$, more succinic and less lactic acid is detected.

Two species originally as signed to the genus Actinomyces do not produce this same set of products. The organisms originally described as Actinomyces eriksonii produce major amounts of acetic and lactic acids as do bifidobacteria. On the basis of this and other characteristics, other investigators have agreed that this is a species of Bifidobacterium. Actinomyces propionicus produces major amounts of propionic acid and it is now accepted that the species propionicus is not a member of the genus Actinomyces.

The anaerobic corynebacteria and propionibacteria produce propionic and acetic acids as major products with variable amounts of isovaleric, formic, succinic, and lactic acids. This pattern of products is also produced by Veillonella species.

The above results were obtained by liquid-liquid chromatography. However, for these two genera and many of the other groups of anaerobes differentiation or identification can be based on gas-liquid chromatographic analyses because the major products are volatile - (Fig. 2). Gas-liquid chromatography of ether extracts of acidified cultures requires only 2 or 3 minutes of the analyst's time. However, succinic and lactic acids are not detected unless a separate sample is methylated to form volatile derivatives of the succinic and lactic acids.

Ramibacterium alactolyticum, an organism morphologically similar to propionibacteria. produces acetic, butyric, and caproic acids as major products. In some cultures, the corresponding 2, 4, and 6-carbon alcohols and caprylic acid are also produced. 
Some species of the genus Eubacterium which have on superficial bases sometimes been confused with corynebacteria or Actinomyces produce distinctive patterns of butyric and other volatile acids. Other species currently assigned to the genus Eubacterium produce no major amounts of any acid or alcohol.

Species now in the genus Gatenabacterium have distinctive patterns of products. Catenabacterium catenaforme produces lactic acid almost exclusively, but we cannot yet propose that it is truly a species of Lactobacillus because we have evidence that at least some strains pro. duce spores, in which case it is truly a Clostridium.

Among the clostridia there are at least 7 distinct patterns of products ranging from only acetic acid to complex but reproducible mixtures of eight or more products - (Fig. 3). If patterns from lactate or basal medium are also considered, several of the 7 fermentation groups of species can be further subdivided.

The Gram-negative non-sporeforming rods divide into two major groups; those that produce butyric acid as a major product and those that do not - (Fig. 4). The International Taxonomic subcommittee has recommended that butyric acid species be placed in the genus Fusobacterium; This genus can be further subdivided on the basis of production of propionate from threonine, which is also easily tested with gas chromatography.

The non-butyric species are to be placed in the genus Bacteroides. Different patterns of products further divide species in this genus into the melaninogenicus group, the propionic succinic group, and the acetic formic group.

The anaerobic Gram-positive cocci have several distinctive patterns of products, although a number of species produce no major amounts of acids or alcohols.

Acid and alcohol products, and cell wall composition are not comparable bases for differentiation of bacteria. The several species of the genus Lactobacillus can be divided into only two subgroups on the basis of acid products (homofermentative and heterofermentative), while cell wall composition further separates the species in these two subgroups. Much the same picture is true for the other genera examined although the se two independent analyses often point to the same conclusion; for instance, in the assignment of Actinomyces eriksonii to the genus Bifidobacterium, or in the differentiation of Ramibacterium alactolyticum from the propionibacteria - (Table 1). It appears that both characteristics are stable properties of these species, and therefore each is found to be quite uniform among accurately identified strains within species. If one considers only three major groups based on LL-, meso-, or no diaminopimelic acid in the cell wall, the fermentation product patterns are generally more specific. If cell wall sugar or amino acid composition is considered, then cell wall composition is much more specific.

Patterns of alcohol and acid products are extremely helpful in clearcut differentiation of genera or groups of species within genera. In some cases there are distinctive patterns of products that we so far have found in only single species. Of about 4,000 tested strains, we know of only two cases where strains were found on the basis of other available tests to belong to one species yet produced different alcohol 
and acid products. One exception is Clostridium botulinum in which the different alcohol and acid products correlate with the other biochemical properties of each of the toxin types or proteolytic and non-. proteolytic subgroups within the toxin types.

The other exception is Bacteroides melaninogenicus in which saccharolytic strains produce different products from non-saccharolytic strains. These may prove to be distinct species.

Chromatographic analyses may also be made of the amine products of species. The total concentrations of amines produced by many cultures, especially of proteolytic species, are often as great as the concentrations of acid and alcohol products. Fifteen available strains of the proteolytic species Clostridium limosum produced acetic acid as the only acid product. The acetic acid is produced from peptone or protein substrate. Studies of the component cell wall sugars indicated that there were 2 or 3 subgroups in the 16 phenotypically similar strains that we had assigned to that species. When chromatographic analyses were made of the complex amine mixtures produced, two groups were also found. One correlated well with one cell wall group of 13 strains, the other embraced the remaining two cell wall groups of 2 strains and 1 strain each. On the other hand, analyses of the amines produced by the different strains of Propionibacterium acnes discussed by C. S. Cummins and J. L. Johnson, failed to show any difference in the amine pattern of the two subgroups that they each detected.

Thus it is evident that cell wall composition and patterns of major catabolic products are independent properties and may differentiate groups of organisms at entirely different levels of specificity. Since they are both stable characteristics of bacteria, they of course show excellent correlation within each species of subspecies.

\section{REF ERENC ES}

Holdeman, L. V., E. P. Cato and W. E. C. Moore. 1967. Amended description of Ramibacterium a lactolyticum Prevot and Taffanel, with proposal of a neotype strain. Int. J. Syst. Bact. 17:323-341. , and - 1967. Fermentation pathways of anaerobic bacteria. Bull. Off. Int. Epiz. 67:1230-1248.

Moore, W. E. C. and E. P. Cato. 1965. Synonymy of Eubacterium limosum and Butyribacterium rettgeri: Butyribacterium limosum Comb. nov. Int. J. Syst. Bact. 16:69-80. and L. V. Holdeman. 1966. Fermentation pathways of some Clostridium species. Int. J. Syst. Bact. 16:383-415.

Outline of Clinical Methods in Anaerobic Bacteriology. 1968. V.P.I: Anaerobe Laboratory, Dept. of Veterinary Science, Blacksburg, Virginia. 24060 . 